\title{
Summary-Analysis and Literature Review of Trans- Cutaneous Oxygenation on Lower Extremity Wounds
}

\author{
Howard Myles Kimmel*, Jamie Matteo, Christopher Phillips and Perry Williams \\ Podiatrist, Buckeye Foot Care, USA
}

Submission: April 04, 2017; Published: April 24, 2017

*Corresponding author: Howard Myles Kimmel, Buckeye Foot Care, Podiatrist in Brook Park, Cleveland, Ohio, USA, Tel: +1-216-267-0304; Fax: +1-216-267-4932; Email: buckeyefootcare@sbcglobal.net

\begin{abstract}
Oxygen is an essential component of wound healing. The discovery of its critical role in maintaining life and healing properties dates back to the late 1700 's. With the invention of hyperbaric oxygen chamber in 1879 research into the medicinal utilization of oxygen began and continues today. More recently, hyperbaric oxygen therapy has been studied and implemented for a variety of functions including wound healing, but recently its effectiveness has been questioned. Due to some of its more adverse side effects, the development of a more localized therapy option transcutaneous trans-cutaneous oxygenation has found its niche in the role of wound healing. Compiling the research and studies of the use of transcutaneous trans-cutaneous oxygenation in the literature, a statistical analysis was utilized to evaluate its efficacy in wound healing.
\end{abstract}

Keywords: Oxygen; Oxygen Therapy; Topical Hyperbaric oxygen; Ulcer

\section{Introduction}

Wound healing is a complex process of biochemical and cellular events. Anything that disrupts this highly complicated process can possibly cause a wound not to heal. Venous ulcer patients have systemic and local conditions such as venous hypertension that can cause compromised vascularity Wollina et al. [1]. Patients who suffer from arterial ulcers are having an etiology caused by severe macro vascular disease, while diabetic ulcerations are related to both microvascular and macro vascular disease Stephens \& Hunt [2].

Hypoxia can be defined as a decreased level of oxygen. In normal wound healing, there are times in which the wound needs to be hypoxic. Prolong periods of hypoxia are abnormal. Successful wound healing depends on a sufficient blood supply to bring various components including oxygen to help facilitate the wound to heal. The central portion of the wound is the area that is usually most hypoxic, while there more oxygen on the periphery of the wound. The partial pressure of oxygen $\left(\mathrm{pO}_{2}\right)$ in dermal wounds ranges from $0-10 \mathrm{mmHg}$ centrally to $60 \mathrm{mmHg}$ at the periphery. The $\mathrm{pO}_{2}$ in the arterial blood is approximately $100 \mathrm{mmHg}$ Gordillo \& Sen [3].

Angiogenesis, or neovascularization, takes place as preexisting vessels send out capillary buds to produce new vessels.
Proliferating capillaries bring oxygen and micronutrients to growing tissues and remove catabolic waste products. Following an injury, angiogenesis is stimulated by the synergistic interaction of growth factors and cytokines secreted by damaged cells in wound tissues exhibiting hypoxia. Oxygen and its reactive derivative hydrogen peroxide are known to induce angiogenic responses such as the induction of VEGF expression Sen et al. [4], Sen et al. [5], Trabold et al. [6]. There are essential growth factors associated with angiogenesis (BFGF, HB-EGF, KGF and VEG-F) which are increased with oxygenation therapy Kalliainen et al. [7]. $\mathrm{O}_{2}$ treatment induces VEGF mRNA levels in endothelial cells and macrophages and increases VEGF protein in wounds in vivo Sheikh et al. [8]. VEGF was found to have a 20fold increase with $\mathrm{O}_{2}$ therapy Hunt [9]. Chronic hypoxia impairs angiogenesis causing death and dysfunction of tissue Hopf et al. [10]. Administration of topical $\mathrm{O}_{2}$ accelerates vessel growth and alleviates the danger of tissue death Knighton et al. [11].

Lavan etal. [12] illustrated that the rate of synthesis of collagen in closed wounds is accelerated by hyperoxia Lavan and Hunt [12]. Collagen synthesis has multiple post-translational steps that are directly $\mathrm{O}_{2}$ dependent including prolyl hydroxylase, lysyl hydroxylase and lysyl oxidase. Increasing oxygenation results in increased collagen deposition and tensile strength Hunt [13]. It 
has also been observed that $\mathrm{O}_{2}$ may trigger the differentiation of fibroblasts to myofibroblasts which are responsible for wound contraction Roy et al. [14]. The oxygenation of wound tissue is an extremely sensitive indicator of infection in surgical patients Greif et al. [15]. Granulocytes are unable to ingest bacteria without adequate perfusion therefore increasing the bacterial load and increasing the rate of infection. Reactive oxygen species (ROS), such as $\mathrm{H}_{2} \mathrm{O}_{2}$, which are generated by NADPH oxidases in wound neutrophils and macrophages, mediate the ability of supplemental 02 to reduce infection Hopf [16].

In 1775 Priestly made medical history by discovering oxygen and demonstrating that oxygen maintained the vitality of candle light and suggested its important role in human life. However it was Henshaw, a British physician, who introduced the hyperbaric chamber. Further progression of the device led to Fontaine's use of the chamber in the operating room in 1879 Braswell and Crowe [17]. The United States Navy began to use hyperbaric oxygen in 1943 for treatment of decompression sickness and air embolism. In the early 1960s the National Sea Foundation investigated the claims made by Jacques Cousteau that his divers healed their work related wound better when they lived in an under the sea construct at 35 feet under the Red Sea.

In the 1970s the Hyperbaric Oxygen Committee of the Undersea Medical Society defined four categories of clinical usage. Categories I and II demonstrate the value of oxygen in wound healing. Initially large recompression chambers were used, with and without the modification for mask breathing of oxygen. The toxic side effects from the systemic absorption of oxygen including pulmonary and CNS toxic reactions led to a more localized therapy. It was Olejniciak who reported success with the application of high pressure oxygen to injured extremities using trans-cutaneous oxygen Olejniczak and Zielinski [18]. Specially built chambers were designed to fit around the affected limb creating a controlled, sealed pressure atmosphere and therefore limiting systemic exposure.

Hyperbaric Oxygen (HBO) is a treatment in which patients breathe in $100 \%$ oxygen inside a chamber that is pressurized at higher than the sea level pressure of greater than $1 \mathrm{~atm}$. According to Undersea and Hyperbaric Medical Society position paper, in order for the therapy to be officially recognized pressurization should be at least 1.4 atms or higher. Van Unnik found that when treating Clostridial myositis and myonecrosis, gas gangrene, it is essential to achieve tissue $\mathrm{PO}_{2}$ of $250 \mathrm{~mm} /$ Hg Van Unnik [19]. This is necessary to stop the advancing phlegmon caused by infection tissue producing alpha toxin. With compartment syndrome and crush injuries, HBO can be used as an additive therapy to possibly decrease complications that are often associated with these types of injuries. HBO is recommended for crush injuries graded Gustilo IIIB and C fractures Gustilo et al. [20]. Treatment criteria for HBOT in crush injuries varies depending on suspected pathophysiology.
With problematic ulcerations and recurrent wounds, HBO is a recommended as an adjunctive therapy when these wounds have failed other treatments. The suggested treatment protocol in diabetic wounds is HBO given at 2.0 to 2.4 ATA for 90 minutes daily for 4 to 6 weeks Feldmeier et al. [21]. As previously discussed, the toxic side effects associated with HBO have led to the development of a more localized therapy options. One of these is the Trans-Cutaneous Oxygen Therapy. By minimizing the size of the unit, these types of devices have their oxygen provided by traditional respiratory oxygen concentrators. They are easily transportable allowing for application of the treatment at virtually any care site [22].

Trans-Cutaneous Oxygen Therapy (TCOT) is thought to provide increased supplies of superoxide dismutase, catalase, and reduced glutathione and other free radical quenches Gordillo and Sen [3]. Trans-cutaneous oxygen can eliminate bacteria by producing reductive oxygen species Trabold et al. [6]. TCOT has been demonstrated to increase oxygen of hypoxic wound tissues therefore encouraging of angiogenesis, increased collagen production and tensile strength, increased epithelial proliferation, stimulation of fibroblasts, induction resistance to bacteria and abundant granulation tissue formation Heng [23]. TCOT can be beneficial in reducing edema in extremities in addition to increasing venous blood flow and lymphatic drainage from intermittent positive pressures Tawfick \& Sultan [24]. TCOT systems operate by applying cyclical oxygen pressure directly to the wound site within a sealed and humidified environment. This can provide a greater tissue oxygen diffusion gradient and increased tissue oxygenation. The cyclical nature of the pressure also creates a sequential compression effect which helps reduce peripheral edema and stimulates wound site perfusion.

\section{Analysis of Published TCOT Papers}

The following analysis assesses the various studies published in human subjects with TCOT. Upson provides a clinical report, consisting of two case studies, that discusses the effectiveness of trans-cutaneous oxygen for the treatment of ischemic lower extremity ulcerations and preventing amputations of the lower limb Upson [25]. This clinical report also discusses treatment guidelines for improving the quality of wound care for recalcitrant wounds of the lower extremity. Case 1 is a 54 year old male with multiple ulcerations of his left lower extremity for duration of approximately 10 months. After 8 weeks of TCOT ( 5 days/wk., twice daily for 60-90 minutes) there was reported $75 \%, 48 \%$, and $80 \%$ healing of three of the major ulcerations of the lower left extremity. Case 2 was a 69 year old male with a lower left leg compartment fasciotomy that never healed of 8 months duration. After 1 week of TCOT the treatment was discontinued because the ulceration had completely healed. The authors concluded that trans-cutaneous oxygen provides good results and healing rates of recalcitrant ischemic wounds in the lower extremity. 
Heng et al. [23] reported endothelial cell toxicity from a clinical and cellular level in patients treated for prolonged periods of time for leg ulcerations Heng [26]. Six patients with leg ulcers of varying etiologies were treated with TCOT for a range of 5-9 weeks. All patients received 6 hours of therapy per day on 4 consecutive days per week followed by 3 consecutive days of rest. $4 \mathrm{~mm}$ punch biopsies were also taking from all patients and split in half to be examined under light microscopy and electron microscopy. In the first 4 weeks all patients experienced decreased slough formation on the base of the ulcers, growth of abundant vascular granulation tissue and overall decrease in size of the ulcers. In three of the patients at the 8-9 week mark, depressed white areas were seen within the granular/vascular areas that had generated. TCOT was stopped in all three of these patients and all white areas had been reversed within 1-2 weeks of the oxygen therapy cessation. These white areas were assumed to be due to oxygen toxicity.

Under light microscopy biopsies of the white areas showed a paucity of capillaries with fragmented nuclei from neutrophils. Biopsies from controlled leg ulcerations not receiving transcutaneous oxygen showed granulation tissue with fewer capillaries than those biopsies from leg ulcers that had received TCOT. Under electron microscopy, biopsies from the control leg ulceration showed endothelial cells with slight indentation of nuclear membranes and intact mitochondria which appear to be fewer than those in endothelial cells of oxygen treated ulcers. Biopsies from tissues that had been treated with prolonged hyperbaric oxygen therapy (8 weeks or more) showed capillaries lined with endothelial cells with marked indentation of nuclear membranes. Many of the mitochondria of these cells appeared swollen and degenerate. Also, many granules from ruptured neutrophils were found among the damaged endothelial cells. The authors concluded that TCOT can increase the number of capillaries in tissues markedly, however detrimental effects can occur in prolonged oxygen therapy.

Leslie et al. evaluated the use of TCOT for 2 week duration in a randomized controlled trial (RCT) Leslie et al. [27]. There were a total of 29 patients with healing diabetic foot ulcers (12 in the TCOT group and 16 in the control group). After the 2 week RCT the decreasing change in ulcer size did not differ statistically between the TCOT group and the control group. Also, a trend towards slower wound healing was noted in the TCOT group. It was concluded that the healing of diabetic foot ulcers was not accelerated by the use of TCOT in this particular study.

Heng et al. [28] reported a prospective randomized controlled trial to confirm the efficacy of TCOT in stimulating angiogenesis and healing in necrotic and gangrenous wounds Heng [26]. This prospective study looked at a total of 40 patients (79 ulcers) that were collected over a period of 12 months who were randomly divided into either a TCOT treatment group or a standard wound care group. Following sharp debridement the TCOT group was treated 4 hours per day, 4 days per week for 4 weeks. Oxygen pressures always remained between 1.004 and 1.013 atmospheres. The standard wound care group underwent rotation every 2 hours to reduce pressure and other tissue pressure reduction techniques.

Patients with OM underwent IV antibiotics (either Ceftriaxone 1-2g daily and or PO Ciprofloxacin 500mg bid). Ceftriaxone (or Vancomycin) and Gentamycin was given for those patients who were septic. Patients with gangrene or life threatening OM received digital or forefoot amputations when appropriate regardless of the group they are in. Results showed that $90 \%$ of the wounds in the trans-cutaneous oxygen group healed compared to only $22 \%$ of the wounds in the standard wound care group. The size of the ulcers showed that they were statistically smaller in the TCOT group. Capillary density was statistically significantly higher in the TCOT group as well. It was concluded that TCOT is effective in stimulating angiogenesis in the healing of necrotic wounds.

In another study, Heng et al. [28] performed a prospective study that examines the feasibility of using TCOT to heal necrotic wounds Heng et al. [28]. Fifteen patients (24 total necrotic and/or gangrenous ulcerations that either did not improve or worsened after 6 weeks of standard wound care were treated with TCOT. Patients received oxygen therapy 4 hours per day, 4 days consecutive days per week for 4 weeks. This was followed by 2 weeks of rest. If a patient's wound was unhealed after this 6 week course, the therapy procedure was repeated. $100 \%$ of the level 2 wounds healed within 2-4 weeks of oxygen therapy. $90 \%$ of the level 3 wounds healed within 4-10 weeks of treatment. $87.5 \%$ of the level 4 wounds healed within $4-12$ weeks. T-tests showed statically significant decrease in wound size day after day with TCOT. These authors concluded that TCOT is associated with decreased costs as well as improved wound healing.

Landau and Schattner reported a case series evaluating the effects of adjunctive TCOT and low energy laser irradiation on chronic diabetic foot ulcerations that have been refractory to 4.5 to 12 months of wound care treatment Landau and Schattner [29]. The first 100 patients with non-gangrenous ulcerations that had been refractory to at least 14 weeks of convention wound care were evaluated in this study. Oxygen therapy was administered for 150 minutes 2-3 times per week and a He-Ne low-energy laser irradiation was administered concurrently at $632.8 \mathrm{~nm} .5 \mathrm{~mW}$. The laser treatment was administered for 20 minutes at a dose of $4 \mathrm{~J} / \mathrm{cm} 2$ to the face of the ulcer. Patients were monitored every two weeks and results showed an $81 \%$ cure rate after $25 \pm 13$ treatments over $3.2 \pm 1.7$ months. Upon follow up (median 18 months) only 3 of the 81 patients that initially healed had re-ulcerated. The authors concluded that TCOT may be a safe, simple, and inexpensive early adjunctive treatment for patient with chronic diabetic foot ulcerations even though this study was open and uncontrolled.

Edsberg et al. [30] published a prospective uncontrolled case series that evaluates the effects of TCOT with electrical 
stimulation on the healing of chronic wounds Edsberg et al. [30]. All patients (8 total; 4 males, 4 females) were geriatric residents of a long term care facility with stage III or stage IV pressure ulcerations ( 7 sacral and 1 ischial) that were refractory to at least 2 weeks of conventional treatment. TCOT was applied to the wounds of all 8 patients with three of the patients also receiving concurrent electrical stimulation. Oxygen therapy was delivered twice daily for 90 minutes, 7 days a week. Electrical stimulation was administered at 150 volts, 120 pulses/second and $255 \mu$ pulse-pair intervals for 30 minutes once daily, 5 days a week. Healing times ranged from 8-49 weeks. After 4 weeks of treatment, wound size had decreased on average $34.4 \%$. There was no statistically significant increase in healing was noted between the 5 patients that were receiving TCOT alone and the other 3 patients that were receiving the TCOT as well as the concurrent electrical stimulation. The authors conclude that TCOT facilitates healing and full closure of pressure ulcers in patients with and without diabetes mellitus.

Kalliainen et al. [7] performed a retrospective study evaluating trans-cutaneous oxygen an adjunct to wound healing Kalliainen et al. [7] In their study, 7 doctors treated 58 wounds in 32 patients over the course of 9-month-period of time. The wounds were treated with $100 \%$ oxygen that was administered at one atmospheres of pressure for 90 minutes for 4 consecutive days followed by a 3-day rest period. This cycle was repeated as long as the wound showed improvement. Of the 58 wounds, 38 or $65.5 \%$, healed during the treatment period with transcutaneous oxygen. The authors concluded a benefit to wound healing with the application and use of oxygen therapy.

In a case study by Edsberg et al., they evaluated the efficacy of TCOT reducing an epibole on a Stage IV sacral pressure ulcer on a seventy-seven year old female patient Edsberg et al. [30]. The wound was treated with trans-cutaneous oxygen via a disposable sacral unit for 60 minutes, two times a day, seven times per week. Treatment also included a high-volt, pulsed electrical stimulation for 30 minutes a day five times a week for a period of 13 weeks. Full wound closure was obtained at the end of the treatment period.

Gordillo et al. [22], reported that trans-cutaneous oxygenation significantly improved wound size Gordillo et al. [22]. The goals of the study: were to examine the changes in wound closure size and look at the expression of oxygensensitive genes, such as VEGF, in biopsies collected from wound edge tissue. In this study, 1,854 patients were screened and of those 57 patients were enrolled. 32 patients were enrolled in the HBO arm and 25 patients were enrolled in the TCOT arm. In this particular study, HBO did not result in significant improvements in wound closure.

The data analysis to access the efficacy of TCOT was identical to that used for HBO. TCOT in this study was found to have a significant improvement of wound closure by decreasing wound volume. The study then directed their examination to
$\mathrm{O}_{2}$ sensitive genes in the wound edge tissue. They goal was to the presence of three genes, VEGF, TGF- $\beta 1$, and COL1A1. These genes were selected based on their oxygen sensitivity and their functional relevance of the gene products to wound healing. The effect of supplemental oxygen therapy was analyzed by measuring the relative change in the target gene expression for each individual compared with their baseline T0 values. Neither TGF- $\beta 1$ or COL1A1 showed any significant change. VEGF however, was significantly higher in TO-treated healing wounds. Neovascularization is stimulated by hypoxia but cannot sustain it. Hyperoxia can also induce VEGF. This study is the first to demonstrate that TCOT significantly induces VEGF expression in chronic wounds.

Tawfick and Sultan investigated if TCOT offered an improved outcome over conventional compression dressings in the management of refractory venous ulcers Tawfick and Sultan [24]. This study had two goals: one was to study the proportion of ulcers healed at 12 weeks and the second goal was the time taken for full closure, percentage of reduction in the ulcer size at 12 weeks, MRSA elimination, pain reduction, and recurrence rates. Patients were either enrolled in TCOT $(n=46)$ or conventional compressive dressings (CCD) ( $n=37)$ for 12 weeks or until full healing was demonstrated. At 12 weeks, $80 \%$ of the TCOT managed ulcerations were fully healed compared to $35 \%$ of those that were treated with CCD. The average time to complete healing in TCOT was 45 days as compared to 182 in those with CCD. At 12 month follow up none of the ulcerations that were treated with TCOT showed any signs of recurrence.

Blackman et al. [31], investigated the clinical efficacy of a pressurized TCOT on 28 patients in an outpatient setting Blackman et al. [31] Seventeen patients were treated with TCOT five times per week for an hour per treatment in pressure cycles between 5 and $50 \mathrm{~mm} / \mathrm{hg}$. Eleven patients were used as the control arm and treated with silver-containing dressings changed at least twice per week. Fourteen of the 17 ulcers in the treatment group, $82.4 \%$, in the treatment group healed while 5 of the 11 ulcers in the in the control group, $45.5 \%$, healed. The median healing time was 56 days for the treatment arm and 93 days for the control.

Heng published a case study in 1983 discussing the use of TCOT for a foot ulcer in a patient with polyarteritisnodosa Heng [32]. The 63 year old male presented with a large area of necrosis involving the plantar aspect of the left foot. Using a disposable polythene bag TCOT was administered at fifteen liters per minute for six hours per day, for four consecutive days each week, for six weeks in duration. The pressure of the oxygen was maintained at $25-30 \mathrm{mmHg}$ above atmospheric pressure. Three unsuccessful attempts at split thickness skin grafts application were made before a second course of TCOT of four weeks duration was completed. After completion, the ulcer was treated with conservative wound care and healed in six months. During the entire treatment course of the ulceration the patient 
remained on oral prednisone $20 \mathrm{mg}$ and Cytoxan 50mg daily. This case reveals that the use of TCOT in can produce granulation tissue in deep ulceration and result in limb salvage.

In another case study by Heng, two patients with leg ulcers characteristic of pyodermagangrenosum were treated with TCOT Heng [33]. Oxygen therapy was administered at $25-30 \mathrm{mmHg}$ above atmospheric pressure at a rate of about $151 / \mathrm{m}$ for $6-8$ hours per treatment period, 4 times a week for a total of two to four weeks. A total of four ulcers were present at initiation of therapy. The ulcerations varied in size, ulcer 1 measured approximately $5000 \mathrm{~mm} 2$, ulcer 2 approximately $3850 \mathrm{~mm} 2$, ulcer $31000 \mathrm{~mm} 2$, and ulcer $4250 \mathrm{~mm} 2$. The two largest ulcers healed at a rate of $106(2 \%)$ and 108 (2.5\%) mm2/day; the medium ulcer closed at the rate of $33 \mathrm{~mm} 2 /$ day; and the smallest at $5 \mathrm{~mm} 2 /$ day. The most significant finding after the initiation of TCOT was the almost immediate arrest of any further extension of the ulcerations. In the 3 weeks prior to therapy they enlarged at a rate of $473 \mathrm{~mm} 2 /$ day. The two smallest ulcers healed 6 weeks after initiation of therapy and the larger two healed within 12 weeks.

In a similar study Heng et al. used a modified technique for administering hyperbaric oxygen with the use of disposable polyethylene bags in the treatment of arterial leg ulcers Heng et al. [33]. Six patients with a total of twenty-seven chronic arterial ulcers were treated. Five men with a total of ten ulcers served as controls. Eighteen of the twenty-seven treated group ulcers (5/6 patients) were healed within six to twenty-one days. A healing rate of $7.8 \% \pm 1.15 \%$ per day for the healed ulcers was recorded. In addition a $50-90 \%$ reduction in size of seven of the nine remaining ulcers was noted after a three week period. None of the ulcers in the control group healed. The potential advantages of the disposable bag method included fairly low expense, lack of cross-infection, and simplicity in the administration of oxygen. The patients could be treated either inpatient or outpatient as well.

Fries et al. [34] evaluated the wound healing efficacy of topically applied pure oxygen on pigs with dermal excisional wounds Fries et al. [34]. Four female $80 \mathrm{lb}$. pigs were used for this study. Under sedation, dermal wounds (1in x 1in.) were inflicted along the dorsum of each pig using size 10 scalpel. A total of 40 wounds throughout the 4 pigs were studied. Half of the wounds on each pig were subjected to topical oxygen treatments as the other half of the wounds on the same pig were left open to room air. The oxygen treatments were administered for 3 hours per day for 7 days. These authors conclude that topically applied oxygen is capable of oxygenating the superficial wound tissue but not deep tissue and that the topical application of pure oxygen to open wounds had some favorable impact on the overall healing process.

Said et al. hypothesizes that transdermal sustained-delivery oxygen therapy would improve wound healing in an animal model Said et al. [35]. In this animal model, 4 full-thickness $7 \mathrm{~mm}$ punch biopsies were created in each ear of a young female New Zealand white rabbits. Oxygen treated wounds received continuous 100\% Oxygen at $\mathrm{ml} / \mathrm{hr}$. under a semi-occlusive dressing. Ears were harvested each at day 5 and day 8. Using a histological analysis wounds showed significantly greater wound healing at both days 5 and 8 in the oxygen therapy group. Epithelization across wounds was nearly double in the oxygen treated group compared to that of the control group. These authors concluded that epithelial wound healing is improved in an environment of sustained-delivery $100 \%$ oxygen.

Sen discussed the role of oxygen and its effect on wound healing Sen [36]. When attempting to oxygenate a wound it is important to utilize a multifaceted approach to wound healing. Of note, it is important to remember that both too little and well as too much oxygen may impede wound healing. The study suggests that it seems important to assess each wound and dose oxygen appropriate to each individual wound. The author also asserts that the wound tissue does not discriminate between whether the oxygen is delivered topically or systemically.

A literature review by Gordillo and Sen evaluated the evidence based recommendations for the use of TCOT in the treatment of lower extremity wounds Gordillo \& Sen [3] They stress that it is important for practitioners to be mindful of the local wound environment, as this is the ultimate determinant of 02 delivery. Other factors such as infection, blood supply and health of the patient are of the utmost importance also. Transcutaneous oxygenation is another treatment modality available to practitioners managing difficult wounds. Supplementing wound tissue p02 has shown to increase collagen deposition and decrease wound infection Knighton et al. [11]. It has also been shown in the literature review, evidence supporting the role of p02 and angiogenesis.

There are some critics to the use of trans-cutaneous oxygen. Feldmeier et al. [21] made a position statement on the use of topical oxygen for chronic wounds Feldmeier et al. [21]. It was stated that with topical oxygen in an airtight chamber or polyethylene bag is sealed around a limb or trunk, while high flow oxygen is introduced in the chamber/bag over the wound. Pressures of just over 1.0 atmosphere are recommended due to the concern that any higher pressures could potentially decrease arterial/capillary inflow to the tissue in the affected area. The literature on topical oxygen at this point consists mainly of small case series and uncontrolled trials which don't focus specifically on any particular type of wound. The only randomized controlled trial for topical oxygen in diabetic foot ulcers published at that did not demonstrate improved wound healing Leslie et al. [27]. It is noted that for optimal effects from TCOT, wounds must be debrided prior to oxygen treatment. Any exudative or proteinaceous coating over the base of the wound will significantly impair the oxygen penetration. 
Cronje stated a major challenge to researchers is how to improve the delivery of oxygen and blood to wounds that have these deficiencies Cronje [37]. One of the ways to supplement oxygen to deficient tissues that are deficient is through TCOT. The author states that the oxygen absorbed through intact skin is negligible and even through open wounds it is extremely small. Wound healing depends on a gradient that extends from the periphery into the middle of the wound which directs and initiates the normal wound healing process. The author states that even if topical oxygenation increases wound oxygen levels, the gradient would be reversed putting higher levels of oxygen at the periphery of the wound. Systemic hyperbaric oxygen therapy actually accentuates that normal gradient, unlike TCOT.

\section{Experimental Section}

Our initial intent was to do a meta-analysis that compared studies examining the effect of Hyperbaric Oxygen treatment on ulcer size. After reviewing the literature, we found no studies similar enough to do a formal Meta analysis. As an alternative to our initial objective, we decided to use a model-based approach to explore the data. Thus, we used the data reported in the 16 studies were viewed to develop a model describing the functional relationship between the change in ulcer size and the treatment method (oxygen versus control). We searched the 16 studies originally reviewed for similar metrics that provided us data to build this model. Five of these 16 studies reported results that allowed us to estimate a model. They were: Heng et al. [33], Heng et al. [28], Leslie et al. [27], Tawfick and Sultan [24], and Edsberg et al. [38] Heng [23], Leslie et al. [27].

The response variable that we examined was the mean ulcer size. We conditioned our response variable on whether the patients received oxygen treatment and the length of the study. That is, we compared the reduction in ulcer size in treatment groups and control groups and controlled for how long patients were observed. We used a linear mixed effects regression model that allowed the intercept to change based on the study (i.e., it was a random effect). We weighted our model using the sample size in each study. Additionally, in our fixed effects, we forced the intercept to be the same for treatments and controls in ceat time $=0$ the treatment would not affect the size of the ulcer. Thus model was of form: in ulcer size. From these values we calculated the overall change in ulcer size by multiplying the daily increase/decrease by the length of the study. For the treatment group this resulted in a negative ulcer size for the 21day study, indicating that most of the patients healed before the 21day study was concluded. To avoid using impossible negative ulcer size values we selected the number of days in which the ulcer size was estimated to be $\geq$ 0 . This was on day16. Based on these caveats, we recommend that these results be viewed as exploratory, and might not be unbiased estimates of the actual parameter values we attempted

Where yij was the ulcer size of the its study and jth patient, $\beta 0$ was the mean initial ulcer size, $\beta 1$ was the change in ulcer size due for the treatment group and time since the beginning of the study, and $\beta 2$ was the change in ulcer size due to times since the beginning of the study for the control group. Hi was 0 , and variance $\rho$. We assumed the errors were normal, with mean 0 and variance $\sigma 2$. We estimated our model parameters using thelme 4 package in Program $\mathrm{R}$ (function: lmer). We
examined residual plots to determine if we violated our model assumptions.

We note that there was much variability among the study designs, and that this comparison does not account for all the variability. For example, Heng et al. [23] did not use a completely randomized design to assign patients to either control or addition to the variability among treatments, some of the studies did not explicitly report the values we used in this analysis. For example, most studies reported the mean size of ulcers at the onset of their studies. Tawfick and Sultan did not report the
mean size Tawfick and Sultan [24]. Instead, they divided ulcer sizes into 5 groups, and reported the number of patients that size was, we used the median value for each of the five groups and multiplied it by the number of patients in these groups, summed these values, then divided by the total number of patients. Additionally, Heng et al. [23] Did not report the overall decrease in ulcer size for their 21 day study Heng [23].

Instead they reported the expected daily increase/decrease

$$
\eta \mathrm{i} \sim \mathrm{N}(0, \rho)
$$$$
y i j=\beta 0+\beta 1(\text { treatment }) \times(\text { time })+\beta 2(\text { time })+\eta i+E i
$$

Table 1: Table of data used for our model. Study ID1 was from Leslie et al. [27], studyID2 was from Tawfick and Sultan [24], Edsberg et al. [38], study ID7 was Heng et al. [23], study ID8 was Heng et al. [28].

\begin{tabular}{|c|c|c|c|c|c|}
\hline SNO & Study ID & Ulcer size & Day & Sample size \\
\hline 1 & 1.00 & 551.80 & 1.00 & 12.00 \\
\hline 2 & 1.00 & 319.60 & 1.00 & 1.00 \\
\hline 3 & 1.00 & 423.00 & 8.00 & 12.00 \\
\hline 4 & 1.00 & 223.20 & 8.00 & 16.00 \\
\hline 5 & 1.00 & 308.10 & 15.00 & 12.00 \\
\hline 6 & 1.00 & 115.30 & 15.00 & 1.00 \\
\hline
\end{tabular}
to estimate. All of the data used in our analyses are reported in (Table 1) (Figure 1). 


\section{Juniper Online Journal of Orthopedic \& Orthoplastic Surgery}

\begin{tabular}{|c|c|c|c|c|c|}
\hline 7 & 2.00 & 1967.50 & 1.00 & 46.00 & 1.00 \\
\hline 8 & 2.00 & 1978.50 & 1.00 & 37.00 & 0.00 \\
\hline 9 & 2.00 & 78.70 & 85.00 & 46.00 & 1.00 \\
\hline 10 & 2.00 & 771.62 & 85.00 & 37.00 & 0.00 \\
\hline 11 & 3.00 & 30.10 & 1.00 & 8.00 & 1.00 \\
\hline 12 & 3.00 & 19.75 & 29.00 & 8.00 & 1.00 \\
\hline 13 & 7.00 & 334.35 & 1.00 & 6.00 & 0.00 \\
\hline 14 & 7.00 & 180.53 & 1.00 & 5.00 & 1.00 \\
\hline 15 & 7.00 & 0.75 & 17.00 & 5.00 & 0.00 \\
\hline 16 & 7.00 & 185.97 & 17.00 & 12.00 & 0.00 \\
\hline 17 & 8.00 & 270.00 & 1.00 & 12.00 & 1.00 \\
\hline 19 & 8.00 & 1200.00 & 1.00 & 12.00 & 0.00 \\
\hline
\end{tabular}

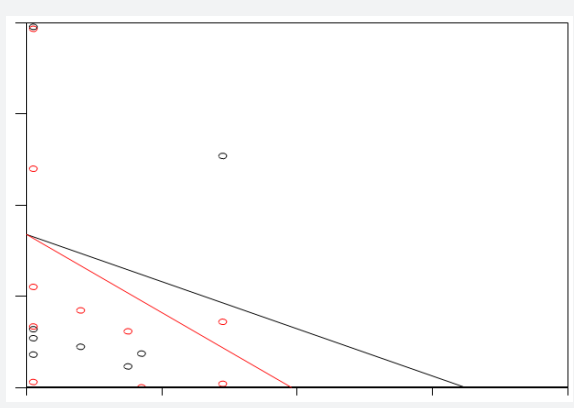

Figure 1: Relationship between ulcer size, no. of days since the onset of the study, and trans-cutaneous oxygen therapy (redline) or standard treatment (blackline) on foot ulcers from 5 studies reported in the literature.

\section{Results and Discussion}

Our model appeared to meet the assumptions of a linear regression model. All of the predictor variables in our model were significant, and P-values were 0 . We found that the treatment group healed faster $(\mathrm{P}=0.000)$ than the control group on average. The fixed effects parameter estimates (and estimated standard errors) were: $\beta 0=839.55(77.82)$, $\beta 1=-12.94(0.60)$, and $\beta 2=-8.49$ (0.66). Thus, the mean ulcer size before the studies began was $839.55 \mathrm{~mm} 2$, and decreased in size by $12.94 \mathrm{~mm} 2$ per day for the control groups, and decreased in size by $12.94+8.49=21.43 \mathrm{~mm} 2$ for the treatment groups. The expected time for 100 percent recovery was 40 days for the treatment group and 65 days for the control group. The standard deviation of our random effect (i.e., $\sqrt{\rho} \rho$ ) was $167.17 \mathrm{~mm}$.

\section{Conclusion}

Multiple studies were evaluated for this research looking at the ability of TCOT in healing chronic wounds that had failed other conservative treatment measures and otherwise had a high possibility of not healing. We did not limit out research to parameters specific wound types, in order to allow us to better assess which type of wounds have been shown to be responsive, or not, to TCOT. In wound care, TCOT appears to provide the benefits of full body hyperbaric oxygen while avoiding the potential systemic adverse effects. The addition of therapeutic oxygen levels to an otherwise hypoxic region of the body helps with leucocyte function, sequesters the otherwise unopposed oxidative free radicals, and allows angiogenesis to continue. The oxygen levels and pressures provided by TCOT establish an ideal environment for these processes to occur.

The efficacy and safety of TCOT in treating chronic ulcerations that have previously failed conventional treatment regimens has been demonstrated within the published literature in numerous RCTs, controlled studies and case reports as being an effective adjunctive wound care modality. It is important to note, that there are only a limited number RCTs performed to date and the methods between the studies thus far are inconsistent. Further RCTs with large sample sizes and consistent study parameters are needed to adequately affirm these positive results to the larger greater patient population. However, this being said, our review suggests that TCOT is likely to be effective in healing recalcitrant ulcerations than both standard conventional therapies and other adjunctive modalities alike.

\section{Acknowledgment}

The authors would like to thank Melanie Johnson, DPM in assisting in the preparation of this manuscripts and also Daphne Slaw ski for researching some of the articles used in the preparation of this manuscript.

Author Contributions Howard Myles Kimmel DPM, MBA was the primary author. Perry Williams performed and wrote the summary analysis section. Jamie Matteo DPM wrote the abstract and the introduction and Christopher Phillips, DPM wrote the analysis of TCOT.

\section{Conflict of Interest}

"The authors declare no conflict of interest". 


\section{References}

1. Wollina U, Birgit Heinig, Luther Kloth (2013) The use of biophysical technologies in chronic wound management. Measurements in Wound Healing. Springer, USA, pp. 313-354.

2. Stephens FO, Hunt TK (1971) Effect of changes in inspired oxygen and carbon dioxide tensions on wound tensile strength: an experimental study. Annals of surgery 173(4): 515-519.

3. Gordillo GM, Sen CK (2009) Evidence-based recommendations for the use of topical oxygen therapy in the treatment of lower extremity wounds. The international journal of lower extremity wounds $8(2)$ : 105-111.

4. Sen CK, Khanna S, Babior BM, Hunt TK, Ellison EC, et al. (2002) Oxidantinduced vascular endothelial growth factor expression in human keratinocytes and cutaneous wound healing. Journal of Biological Chemistry 277(36): 33284-33290.

5. Sen CK, Khanna S, Venojarvi M, Trikha P, Ellison EC, et al. (2002) Copper-induced vascular endothelial growth factor expression and wound healing. Am J Physiol Heart Circ Physiol 282(5): H1821-H1827.

6. Trabold O, Wagner S, Wicke C, Scheuenstuhl H, Hussain MZ, et al. (2003) Lactate and oxygen constitute a fundamental regulatory mechanism in wound healing. Wound Repair Regen 11(6): 504-509.

7. Kalliainen LK, Gordillo GM, Schlanger R, Sen CK (2003) Topical oxygen as an adjunct to wound healing: a clinical case series. Pathophysiology 9(2): 81-87.

8. Sheikh AY, Gibson JJ, Rollins MD, Hopf HW, Hussain Z, et al. (2000) Effect of hyperoxia on vascular endothelial growth factor levels in a wound model. Archives of Surgery 135(11): 1293-1297.

9. Hunt TK (1988) The physiology of wound healing. Annals of emergency medicine 17(12): 1265-1273.

10. Hopf HW, Kelly, Matthew Patrick, Shapshak D (2008) Oxygen and the basic mechanisms of wound healing. Physiology and Medicine of Hyperbaric Oxygen Therapy, USA, pp. 203.

11. Knighton DR, Silver IA, Hunt TK (1981) Regulation of woundhealing angiogenesis-effect of oxygen gradients and inspired oxygen concentration. Surgery 90(2): 262-270.

12. Lavan F, Hunt T (1990) Oxygen and wound healing. Clinics in plastic surgery 17(3): 463-472.

13. Hunt T, Pai MP (1972) The effect of varying ambient oxygen tensions on wound metabolism and collagen synthesis. Surg Gynecol Obstet 135(4): 561-567.

14. Roy S, Khanna S, Wallace WA, Lappalainen J, Rink C, et al. (2003) Characterization of perceived hyperoxia in isolated primary cardiac fibroblasts and in the reoxygenated heart. Journal of Biological Chemistry 278(47): 47129-47135.

15. Greif R, Akca O, Horn EP, Kurz A, Sessler DI, et al. (2000) Supplemental perioperative oxygen to reduce the incidence of surgical-wound infection. New England Journal of Medicine 342(3): 161-167.

16. Hopf HW (2010) Patient Factors as a Major Determinant of Wound Outcome and Infection After Surgery. Hernia Repair Sequelae, Springer, USA, p. 87-95.

17. Braswell C, Crowe DT (2012) Hyperbaric oxygen therapy. Compendium 34(3): E1-E6.

18. Olejniczak S, Zielinski A (1976) Topical oxygen promotes healing of leg ulcers. Medical times 104(12): 114-121.

19. Van Unnik AJ (1965) Inhibition of toxin production in Clostridium perfringens in vitro by hyperbaric oxygen. Antonie Van Leeuwenhoek 31(1): 181-186.
20. Gustilo RB (1990) The management of open fractures. The Journal of bone and joint surgery American 72(2): 299.

21. Feldmeier J, Hopf HW, Warriner RA, Fife CE, Gesell LB, et al. (2005) UHMS position statement: topical oxygen for chronic wounds. Undersea Hyperb Med 32(3): 157-168.

22. Gordillo GM, Roy S, Khanna S, Schlanger R, Khandelwal S, et al. (2008) Topical oxygen therapy induces vascular endothelial growth factor expression and improves closure of clinically presented chronic wounds. Clin Exp Pharmacol Physiol 35(8): 957-964.

23. Heng MC, Pilgrim JP, Beck FW (1984) A simplified hyperbaric oxygen technique for leg ulcers. Arch dermatol 120(5): 640-645.

24. Tawfick W, Sultan S (2009) Does Topical Wound Oxygen (TWO< sub> $2</$ sub $>$ ) Offer an Improved Outcome Over Conventional Compression Dressings (CCD) in the Management of Refractory Venous Ulcers (RVU)? A Parallel Observational Comparative Study. Eur J Endovas surg 38(1): 125-132.

25. Upson AV (1986) Topical Hyperbaric Oxygenation in the Treatment of Recalcitrant Open Wounds A Clinical Report. Physical therapy 66(9): 1408-1412.

26. Heng MC (1993) Topical hyperbaric therapy for problem skin wounds. The Journal of dermatologic surgery and oncology 19(8): 784-793.

27. Leslie CA, Sapico FL, Ginunas VJ, Adkins RH (1988) Randomized controlled trial of topical hyperbaric oxygen for treatment of diabetic foot ulcers. Diabetes Care 11(2): 111-115.

28. Heng MC, Harker J, Bardakjian VB, Ayvazian H (2000) Enhanced healing and cost-effectiveness of low-pressure oxygen therapy in healing necrotic wounds: a feasibility study of technology transfer.

Ostomy Wound Manage 46(3): 52-62.

29. Landau Z, Schattner A (2001) Topical hyperbaric oxygen and low energy laser therapy for chronic diabetic foot ulcers resistant to conventional treatment. Yale J of Biol Med 74(2): 95-100.

30. Edsberg L, Brogan S, Jaynes CD, Fries K (2002) Reducing epibole using topical hyperbaric oxygen and electrical stimulation. Ostomy wound manage 48(4): 26-29.

31. Blackman E, Moore C, Hayatt J, Railton R, Frye C (2010) Topical wound oxygen therapy in the treatment of severe diabetic foot ulcers: a prospective controlled study. Ostomy wound manage 56(6): 24-31.

32. Heng MC (1983) Hyperbaric oxygen therapy for a foot ulcer in a patient with polyarteritis nodosa. Australas J Dermatol 24(3): 105-108.

33. Heng MC (1984) Hyperbaric oxygen therapy for pyoderma gangrenosum. Australian and New Zealand journal of medicine 14(5): 618-621.

34. Fries RB, Wallace WA, Roy S, Kuppusamy P, Bergdall V, et al. (2005) Dermal excisional wound healing in pigs following treatment with topically applied pure oxygen. Mutat Res 579(1-2): 172-181.

35. Said HK, Hijjawi J, Roy N, Mogford J, Mustoe T (2005) Transdermal sustained-delivery oxygen improves epithelial healing in a rabbit ear wound model. Arch Surg 140(10): 998-1004.

36. Sen CK (2009) Wound healing essentials: let there be oxygen. Wound Repair Regen 17(1): 1-18.

37. Cronje FJ (2005) Oxygen therapy and wound healing-topical oxygen is not hyperbaric oxygen therapy: clinical practice: SAMJ forum. South African Medical Journal 95(11): 840.

38. Edsberg LE, Brogan MS, Jaynes CD, Fries K (2002) Topical hyperbaric oxygen and electrical stimulation: exploring potential synergy. Ostomy wound manage 48(11): 42-50. 
Your next submission with Juniper Publishers will reach you the below assets

- Quality Editorial service

- Swift Peer Review

- Reprints availability

- E-prints Service

- Manuscript Podcast for convenient understanding

- Global attainment for your research

- Manuscript accessibility in different formats ( Pdf, E-pub, Full Text, Audio)

- Unceasing customer service

Track the below URL for one-step submission https://juniperpublishers.com/online-submission.php 